\title{
Reporte de dos casos clínicos de adolescentes con asma grave eosinofílica refractaria no controlada tratados con mepolizumab a un año de seguimiento
}

\author{
Report of two clinical cases of adolescents with severe uncontrolled refractory \\ eosinophilic asthma treated with mepolizumab at one-year follow-up
}

\author{
Saraid Cerda-Reyes, ${ }^{*}$ Juan Gabriel Maldonado-Hernández, ${ }^{*}$ Rosa Isela Campos-Gutiérrez, ${ }^{*}$ \\ Nidia Karen Castillón-Benavides, ${ }^{*}$ Armando Partida-Gaytán ${ }^{\ddagger}$ \\ * Servicio de Alergia, Unidad de Especialidades Médicas, Secretaría de la Defensa Nacional, Naucalpan, Estado de México. \\ ‡ Fundación Mexicana para Niñas y Niños con Inmunodeficiencias Primarias A.C., Ciudad de México.
}

Citar como: Cerda-Reyes S, Maldonado-Hernández JG, Campos-Gutiérrez RI, Castillón-Benavides NK, Partida-Gaytán A. Reporte de dos casos clínicos de adolescentes con asma grave eosinofílica refractaria no controlada tratados con mepolizumab a un año de seguimiento. Alergia Asma Inmunol Pediatr. 2021; 30 (3): 104-109. https://dx.doi.org/10.35366/102984

\section{RESUMEN}

Introducción: Aunque el asma grave afecta a menos de $5 \%$ de los pacientes asmáticos, siempre representa un reto diagnóstico y terapéutico. Más de dos terceras partes de los pacientes con asma grave tienen evidencia de inflamación eosinofílica como mecanismo principal detrás de esa gravedad clínica. Recientemente un nuevo anticuerpo monoclonal humanizado anti-IL5 ha sido aprobado en México para el tratamiento de pacientes con asma grave eosinofílica refractaria no controlada a partir de los 12 años de edad. Caso clínico: Compartimos nuestra experiencia clínica inicial con mepolizumab a través de dos casos clínicos de adolescentes con diagnóstico de asma grave eosinofílica refractaria que han recibido tratamiento con mepolizumab 100 mg por vía subcutánea cada 30 días complementario a tratamiento estándar inhalado. Incluimos la descripción clínica que llevó a la decisión de iniciar mepolizumab y comparaciones descriptivas de diferentes variables clínicas (número de exacerbaciones, puntaje de prueba de control de asma, frecuencia de uso de medicamentos de rescate, dosis de medicamentos de mantenimiento, cambios en parámetros de función pulmonar y frecuencia de eventos adversos reportados), y biomarcadores específicos de asma grave (eosinófilos, fracción exhalada de óxido nítrico) antes y después de iniciar mepolizumab a un año de seguimiento. Conclusión: Nuestra experiencia clínica inicial con mepolizumab en el tratamiento de dos adolescentes con asma grave eosinofílica refractaria no controlada ha sido favorable con reducciones importantes en las exacerbaciones de asma,

\section{ABSTRACT}

Introduction: Although severe asthma affects less than 5\% of asthmatic patients, it always represents a diagnostic and therapeutic challenge. More than two-thirds of patients with severe asthma have evidence of eosinophilic inflammation as the main mechanism behind this clinical severity. Recently, a new humanized anti-IL5 monoclonal antibody has been approved in Mexico for the treatment of patients with severe, uncontrolled refractory eosinophilic asthma from 12 years of age. Clinical case: We share our initial clinical experience with mepolizumab through two clinical cases of adolescents with a diagnosis of severe refractory eosinophilic asthma who have received treatment with mepolizumab $100 \mathrm{mg}$ subcutaneously every 30 days complementary to standard inhaled treatment. We include the clinical description that led to the decision to start mepolizumab and descriptive comparisons of different clinical variables (number of exacerbations, asthma control test score, frequency of use of rescue medications, dose of maintenance medications, changes in parameters of lung function, frequency of reported adverse events), and specific biomarkers of severe asthma (eosinophils, exhaled fraction of nitric oxide) before and after starting mepolizumab at 1 year of followup. Conclusion: our initial clinical experience with mepolizumab in the treatment of two adolescents with severe uncontrolled refractory eosinophilic asthma has been favorable with significant reductions in asthma exacerbations, improvement in asthma control, decrease in the frequency of use of rescue medications and improvement in lung 
mejoría del control del asma, disminución en la frecuencia de uso de medicamentos de rescate y mejoría en la función pulmonar de ambos pacientes. Esta experiencia inicial es un panorama que requiere ampliarse a más pacientes y por más tiempo para aportar evidencias de consistencia de resultados observados en otras poblaciones al contexto de pacientes mexicanos con asma grave.

Palabras clave: Asma grave eosinofílica, mepolizumab, anti-IL5. function in both patients. This initial experience is a panorama that needs to be expanded to more patients and for a longer time to provide evidence of consistency of results observed in other populations in the context of Mexican patients with severe asthma.

Keywords: Severe eosinophilic asthma, mepolizumab, anti-IL5.

\section{INTRODUCCIÓN}

El asma es una enfermedad crónica a inflamatoria de la vía respiratoria inferior que se caracteriza clínicamente por tos, dificultad para respirar, sibilancias, sensación de opresión torácica y funcionalmente por obstrucción reversible del flujo de aire a través del árbol bronquial. ${ }^{1}$ Estas manifestaciones suelen ser variables en intensidad, duración y temporalidad, y es frecuente que se reconozcan factores ambientales como detonantes y/o exacerbantes de los síntomas. ${ }^{1}$ En nuestro país aproximadamente $7 \%$ de la población padece asma. ${ }^{2}$ Afortunadamente, tras el diagnóstico e inicio de tratamiento específico y correcto, ${ }^{3}$ la gran mayoría suelen mejorar significativamente su estado de salud y retomar una vida normal. ${ }^{1}$ Sin embargo, entre 4 y $10 \%$ de los pacientes asmáticos esto no ocurre, y padecen de una enfermedad que, a pesar de un tratamiento correcto y optimizado, continúan teniendo afectaciones importantes en su funcionalidad y calidad de vida. ${ }^{4}$

Para estos casos, padecer asma significa tener síntomas que ocurren todos los días, incluso varias veces al día, afección de la cantidad y calidad de su sueño por despertares nocturnos relacionados a síntomas de asma, limitación en funcionalidad y capacidad física, y que incluso se asocia con frecuencia a exacerbaciones que requieren visitas a urgencias, hospitalizaciones e incluso mortalidad..$^{5-7}$

Algunos tratamientos innovadores han modificado las opciones que podemos ofrecer a pacientes con asma grave. ${ }^{8}$

La evaluación especializada nos permite conocer las vías de señalización involucradas y subyacentes a esa gravedad. En la actualidad, en México podemos inhibir con alta precisión, especificidad y eficacia clínica estas vías de señalización con al menos cuatro anticuerpos monoclonales disponibles. ${ }^{8}$

Se ha estimado que hasta $79 \%$ de los pacientes con asma grave tienen evidencia de inflamación eosinofílica y este mecanismo fisiopatogénico representa el endotipo inflamatorio más frecuente del asma grave., ${ }^{9,10}$

La interleucina 5 (IL-5) participa en la proliferación, maduración, activación y supervivencia de los eosinófilos. ${ }^{11} \mathrm{La}$ inhibición de IL-5 con mepolizumab ha sido ampliamente estudiada en múltiples ensayos clínicos, documentando eficacia, seguridad y efectividad para disminuir la inflamación eosinofílica de pacientes con asma grave eosinofílica y otras enfermedades que comparten ese mecanismo fisiopatogénico (rinosinusitis crónica con pólipos nasales, síndromes hipereosinofílicos, poliangitis granulomatosa con eosinofilia, etcétera $)^{12}$ mepolizumab es un anticuerpo monoclonal recombinante humanizado que se une con alta afinidad y especificidad a IL-5, y aunque el mecanismo de acción de mepolizumab no ha sido completamente descrito, bloquear el efecto biológico de IL-5 se asocia con disminución de la inflamación eosinofílica. ${ }^{13}$

Mepolizumab ha demostrado seguridad, eficacia y efectividad clínica para reducir las exacerbaciones de pacientes con asma grave eosinofílica, así como disminuir o eliminar la exposición a esteroides sistémicos en casos con asma grave eosinofílica corticodependiente, mejorar significativamente el control del asma, la calidad de vida y la función pulmonar. ${ }^{14-17}$

Aunque en otros países mepolizumab cuenta con aprobación a partir de los seis años de edad, en México está aprobado como tratamiento complementario a tratamiento estándar en pacientes con asma grave eosinofílica refractaria no controlada a partir de los 12 años de edad. ${ }^{18}$

En la experiencia clínica del Servicio de Alergia de nuestra institución, atendemos alrededor de 3,120 casos con asma al año, y como centro de referencia y concentración evaluamos, diagnosticamos, tratamos y damos seguimiento a pacientes con asma grave.

El objetivo de este estudio fue describir la evolución a un año de tratamiento con mepolizumab de dos sujetos adolescentes con asma grave eosinofílica refractaria no controlada.

\section{PRESENTACIÓN DEL CASO}

Caso 1: masculino de 15 años originario y residente de Guadalajara, Jalisco, con antecedente familiar de hermana con rinitis alérgica. El paciente inició desde los cuatro años con tos nocturna frecuente ( $\geq 3$ veces por semana) y eventos recurrentes de sibilancias sin relación a cuadros infecciosos. Inicialmente fue manejado con broncodilatador $\beta 2$-agonista de acción corta por razón necesaria. En 2013 a la edad de ocho años fue referido a nuestro servicio 
por la persistencia de síntomas diarios caracterizados por obstrucción nasal 5/7, prurito nasal 6/7, prurito ocular 0/7, estornudos 5/7, rinorrea 4/7, tos en accesos largos, eventos de sibilancias y dificultad respiratoria que se incrementaban con la actividad física y por las noches, limitando su capacidad física. La exploración física inicial documentó estigmas de atopia con pliegue Dennie-Morgan, ojeras hiperpigmentadas, cornetes hipertróficos, mucosa nasal edematosa, puentes de moco hialinos y junto con resultados de evaluación paraclínica se documentaron los diagnósticos de rinitis alérgica moderada-grave persistente y asma alérgica no controlada (Tabla 1). Se inició tratamiento con budesónida 64 g/día intranasal, budesónida-formoterol en dispositivo de polvo seco inhalable dosis media presurizado 160-4.5 $\mu \mathrm{g}$ dos veces al día, montelukast tabletas 5 mg una vez al día, salbutamol en dispositivo de inhalación de dosis media presurizado (IDMp) $200 \mu$ g por razón necesaria e inmunoterapia alérgeno específica (ITAE) subcutánea en esquema convencional para Dermatophagoides farinae y pteronyssinus en mezcla 50:50.

A pesar de buena adherencia al tratamiento y adecuada técnica en el uso de los dispositivos de inhalación, continuó presentando exacerbaciones de asma que requirieron ciclos cortos de esteroide sistémico oral (CSO) (prednisona $1 \mathrm{mg} / \mathrm{kg} /$ día por cinco días), por lo que se incrementó a dosis altas de budesónida-formoterol (320/9 $\mu \mathrm{g} /$ dos veces al día). Por pobre respuesta al tratamiento en 2015, se agregó anticuerpo monoclonal anti-IgE (omalizumab) 300 mg por vía subcutánea cada 30 días con adecuada tolerancia y buena respuesta clínica. Durante un año se mantuvo sin exacerbaciones y con buen control del asma (ACT 25 puntos), permitiendo disminución de tratamiento inhalado a budesónida $100 \mu \mathrm{g}$ dos veces al día y continuando con ITAE.

Un año después, en 2017 volvió a presentar exacerbación relacionada a exposición de grandes cantidades de polvo, por lo que se reinició terapia combinada con budesónida-formoterol, con lo que mostró buena respuesta clínica, manteniéndose asintomático por el siguiente año, lo que permitió disminuir dosis de omalizumab a $150 \mathrm{mg}$ cada 30 días y de budesónida a $100 \mu \mathrm{g} /$ dos veces al día. Los dos años siguientes presentó una exacerbación por año, ameritando ciclos cortos CSO e incremento a dosis altas de budesónida $840 \mu \mathrm{g} /$ día, formoterol $18 \mu \mathrm{g}$ al día. Se repitió evaluación extendida (Tabla 1) y se incrementó dosis de omalizumab 300 mg cada 30 días. A pesar del ajuste, continuó presentando nuevas exacerbaciones que requirieron ciclos cortos de CSO, por lo que se agregó anticolinérgico inhalado (tiotropio a $18 \mu \mathrm{g}$ al día) y se solicitaron nuevos estudios (Tabla 1). En junio de 2020, al documentar persistencia de exacerbaciones y patrón obstructivo en espirometría a pesar de tratamiento con dosis altas de esteroide inhalado asociado a segundo controlador (ICS/ LABA), e incluso tercer controlador (tiotropio), anticuerpo

Tabla 1: Variables clínicas relevantes del caso 1.

\begin{tabular}{|c|c|c|c|c|c|}
\hline Parámetro & 2013 (diciembre ) & 2017 (noviembre) & 2020 (junio) & 2021 (febrero) & 2021 (junio) \\
\hline ACT & 12 & 16 & 8 & 25 & 25 \\
\hline ACQ-5 & 2 & 1.8 & 3.4 & 0.2 & 0.2 \\
\hline $\begin{array}{l}\text { Número de exacerbaciones en } 12 \\
\text { meses previos }\end{array}$ & 2 & 1 & 3 & 0 & 0 \\
\hline Eosinófilos (células), n (\%) & $296(4.0)$ & $230(2.8)$ & $1,330(13.8)$ & $310(2.9)$ & Eos $215(2.5)$ \\
\hline Inmunoglobulina E (Ul/mL) & 542 & 224 & 216 & 122 & 133 \\
\hline \multicolumn{6}{|l|}{$\begin{array}{l}\text { Espirometría basal y } \\
\text { postbroncodilatador }\end{array}$} \\
\hline \multicolumn{6}{|l|}{ Basal } \\
\hline CVF, mL (predicho \%) & $2,545(83)$ & $2,800(86)$ & $2,840(84)$ & $3,860(104)$ & $3,390(92)$ \\
\hline VEF1, mL (predicho \%) & $2,045(75)$ & $1,900(70)$ & $2,020(74)$ & $3,050(99)$ & $3,150(95)$ \\
\hline VEF1/CVF & 0.80 & 0.67 & 0.75 & 0.92 & 0.92 \\
\hline $\mathrm{PEF}(\mathrm{mL} / \mathrm{s})$ & 3,120 & 3,090 & 3,244 & 4,740 & 4,520 \\
\hline \multicolumn{6}{|l|}{ Post-BD } \\
\hline VEF1 (mL) & 2,263 & 2,117 & 2,500 & 3,360 & 3,315 \\
\hline Reversibilidad (\% + mL) & $10.6+218$ & $11.4+217$ & $14+480$ & $10+310$ & $5+165$ \\
\hline \multicolumn{6}{|l|}{ Pruebas cutáneas } \\
\hline Dermatophagoides farinae & 10 & 7 & 6 & & 5 \\
\hline Dermatophagoides pteronyssinus & 12 & 6 & 6 & & 6 \\
\hline Control positivo & 8 & 6 & 6 & & 6 \\
\hline Control negativo & 1 & 1 & 1 & & 1 \\
\hline
\end{tabular}

$\mathrm{ACT}=$ prueba de control de asma, $\mathrm{ACQ} 5$ = cuestionario de control de asma, $\mathrm{VCF}$ = capacidad vital forzada, VEF1 = volumen espiratorio forzado en el primer segundo, y conciente VEF1/VCF. 
Tabla 2: Variables clínicas relevantes del caso 2.

\begin{tabular}{|c|c|c|c|c|c|}
\hline Parámetro & 2014 (agosto) & 2016 (marzo) & 2020 (junio) & 2020 (noviembre) & 2021 (junio) \\
\hline ACQ-5 & 3.4 & 3 & 4.8 & 0.8 & 1 \\
\hline Eosinófilos células, n (\%) & $164(2.0)$ & $400(6.0)$ & $1,250(9.1)$ & $50(0.7)$ & $60(0.9)$ \\
\hline Inmunoglobulina E (UI/mL) & 8.0 & 97.7 & 18.0 & 30.0 & 20.0 \\
\hline CVF, mL (predicho \%) & $3,790(82)$ & $3,580(80)$ & $2,640(59)$ & $4,070(93)$ & $4,080(95)$ \\
\hline VEF1, mL (predicho \%) & $2,900(72)$ & $3,189(79)$ & $2,340(58)$ & $3,390(91)$ & $3,995(96)$ \\
\hline VEF1/CVF & 0.76 & 0.89 & 0.88 & 0.83 & 0.95 \\
\hline PEF, mLs (\%) & $3,290(39)$ & $3,990(45)$ & $4,610(53)$ & $5,670(71)$ & $6,070(77)$ \\
\hline
\end{tabular}

$\mathrm{ACT}=$ prueba de control de asma, $\mathrm{ACQ} 5$ = cuestionario de control de asma, VCF = capacidad vital forzada, VEF1 = volumen espiratorio forzado en el primer segundo, y conciente VEF1/VCF.

anti-IgE (omalizumab) y conteo de eosinófilos $\geq 150$ células/ $\mathrm{mcL}$, se decidió cambio de omalizumab a mepolizumab 100 mg por vía subcutánea cada 30 días. En el seguimiento a los ocho meses y más recientemente a los 12 meses de tratamiento el paciente ha mostrado una respuesta clínica favorable (Tabla 1) con ausencia de exacerbaciones, control del asma, disminución de eosinófilos, IgE y FeNO, con espirometría normal y modificación significativa tras broncodilatador. Se ha logrado disminuir tratamiento a budesónida $100 \mu$ g cada 12 horas, montelukast 10 mg vía oral cada 24 horas y continúa con inmunoterapia con alergoide monomérico 50\% Dermatophagoides pteronyssinus + 50\% Dermatophagoides farinae y mepolizumab $100 \mathrm{mg}$ por vía subcutánea cada 30 días.

Caso 2: se trata de masculino de 15 años con antecedentes neonatales relevantes por prematurez y síndrome de aspiración de meconio e intubación por siete días. A los tres meses por evento de neumonía por broncoaspiración requirió ingreso a terapia intensiva y apoyo mecánico de la ventilación por un mes, con secuelas de compromiso neurológico. Como lactante y preescolar cursó con alergia alimentaria, con manifestaciones gastrointestinales y se documentó histología con hiperplasia linfoide y duodenitis nodular. Fue manejado con dieta de exclusión a proteínas de leche de vaca y huevo por dos años con mejoría y control de los síntomas gastrointestinales, después se pudo reincorporar a una dieta abierta y normal.

Fue referido a nuestro hospital a la edad de ocho años y valorado por Neumología Pediátrica por la historia de tres años de evolución con cuadros recurrentes de tos crónica húmeda, cianosante, no emetizante, ni disnei- zante, en ocasiones inducida por ejercicio con dificultad respiratoria y sibilancias. Además, contaba con historia de rinorrea hialina con ronquido ocasional, que empeoraban con el frío y cambios de estación. Se realizó espirometría que documentó obstrucción moderada con respuesta significativa al broncodilatador y se dejó tratamiento con budesónida-formoterol en dispositivo de polvo seco inhalable dosis media presurizado de 320/9 $\mu$ g cada 12 horas, y salbutamol $200 \mu \mathrm{g}$ por razón necesaria y se refirió a nuestro servicio para evaluación de sensibilizaciones alérgicas y complementación terapéutica. En pruebas cutáneas, no se documentaron sensibilizaciones alérgicas, y tampoco hubo hallazgos relevantes en la cuantificación de IgE ni en biometría hemática (Tabla 2). Persistió con eventos de tos, se clasificó como asma parcialmente controlada, se incrementó la dosis de budesónida $840 \mu \mathrm{g} /$ formoterol 18 $\mu \mathrm{g}$ al día y se agregó montelukast $5 \mathrm{mg}$ una vez al día. En seguimiento se reevaluó y se documentó incremento en IgE, eosinófilos y reversibilidad significativa en espirometría basal y postbroncodilatador (Tabla 2), con lo que se incrementó a dosis altas de budesónida 1,040 $\mu \mathrm{g} /$ formoterol $18 \mu \mathrm{g}$ al día.

Durante los siguientes dos años, a pesar de tratamiento con dosis altas de esteroide inhalado junto con broncodilatador de acción prolongada, antileucotrienos y anticolinérgico (budesónida 1,040 $\mu \mathrm{g} /$ formoterol $18 \mu \mathrm{g}$ al día + tiotropio $18 \mu \mathrm{g}$ al día + montelukast 10 mg una vez al día), presentó exacerbaciones que requirieron ciclos cortos de CSO, por lo que se inició protocolo de estudio de asma grave con fenotipo eosinofílico (Tabla 2). En junio de 2020, a la edad de 15 años se agregó mepolizumab 100 
mg por vía subcutánea cada 30 días, mostrando mejoría significativa del control del asma desde la primera dosis. Como evento adverso, se reportó cefalea a los 60 minutos después de la primera aplicación que se trató con paracetamol 500 mg dosis única y a partir de la segunda dosis en adelante se dejó premedicación con paracetamol 500 mg una hora antes de la aplicación. En último seguimiento tras 12 meses de tratamiento, ha mostrado respuesta clínica favorable, sin exacerbaciones, con buen control del asma, mejor calidad de vida y ha permitido disminuir dosis de tratamiento inhalado, en la actualidad con budesónida $400 \mu \mathrm{g}$ cada 12 horas y mepolizumab 100 mg por vía subcutánea cada 30 días (Tabla 2).

\section{DISCUSIÓN}

El asma grave afecta aproximadamente a $4.5 \%$ de los niños. ${ }^{18}$ El omalizumab, un anticuerpo monoclonal humanizado que neutraliza la IgE, fue el primer anticuerpo monoclonal introducido en 2003 como tratamiento para el asma en casos que no podían controlar su enfermedad a pesar de dosis altas de corticosteroides inhalados (CSI). ${ }^{19}$

Recientemente nuevos anticuerpos monoclonales han empezado a estar disponibles en nuestro país, y nos han permitido incrementar las opciones de tratamiento, que aunque poco frecuentes, siempre representan un importante impacto en la vida de los pacientes y sus familiares, así como retos importantes para los médicos que los atendemos.

La mayoría de los estudios de eficacia y seguridad de los anticuerpos monoclonales para el tratamiento de asma grave representan poblaciones de adultos. Las evidencias para grupos etarios menores (adolescentes y escolares) son considerablemente menores. Específicamente en los estudios fase III de mepolizumab se estudiaron a 34 adolescentes en comparación con 1,841 adultos $\geq 18$ años. ${ }^{20}$

Consideramos que esta experiencia inicial con dos pacientes adolescentes mexicanos es de interés, pues confirma resultados descritos en otras poblaciones, en un grupo etario donde las evidencias no son abundantes y en el contexto clínico de nuestro país.

En centros como el nuestro, donde se refieren y concentran pacientes con asma y asma grave, cada vez es más importante contar con más y mejores herramientas diagnósticas y terapéuticas para caracterizar mejor los casos y permitir estrategias de tratamiento más eficaces, seguras y precisas. ${ }^{21}$

Los anticuerpos monoclonales son una de las estrategias de tratamiento más importantes para asma grave, permitiendo reducir el número de exacerbaciones, mejorar el control del asma, la calidad de vida y disminuir la exposición a CSO. ${ }^{22}$
Recientemente se ha descrito un empalme importante de los criterios de elegibilidad para los distintos anticuerpos monoclonales para el tratamiento de asma grave. ${ }^{1}$ En un estudio español se describió que $>57 \%$ de los pacientes con asma grave cumplen criterios para ser tratados con cualquiera de los biológicos disponibles en ese país (i.e. omalizumab, mepolizumab, reslizumab, benralizumab y dupilumab). Hasta ahora, no existe ningún ensayo clínico que haya comparado formalmente la eficacia y seguridad entre dos o más anticuerpos monoclonales, por lo que las decisiones basadas en la evidencia requieren un análisis individual de cada caso. Además, de manera interesante y como uno de los casos que presentamos, este empalme junto con el seguimiento estrecho de nuestros pacientes puede ofrecernos oportunidades de mejorar el tratamiento ante la falla terapéutica o respuesta parcial a un anticuerpo monoclonal y cambiarlo por otro. ${ }^{23-26}$

Nuestros dos casos clínicos representan los dos fenotipos más frecuentes del asma grave. Uno contaba con antecedente familiar positivo para atopia, diagnóstico de rinitis alérgica, elevación de IgE total, sensibilización alérgica positiva a aeroalérgeno perenne (Dermatophagoides spp.) y cuyo asma no logró controlarse a pesar de tratamiento estándar de alta intensidad (ICS/LABA/LAMA + LTRA). Este caso correspondió a un paciente con asma grave eosinofílica alérgica refractaria no controlada, que incluso con omalizumab continuó presentando exacerbaciones. El segundo caso, al no documentarse sensibilizaciones alérgicas relevantes, correspondió a asma grave eosinofílica no alérgica. Al documentarse concentraciones de eosinófilos $\geq 150$ células/microlitro en ambos, fueron candidatos a mepolizumab.

Este reporte de nuestra experiencia temprana describe hallazgos similares a lo que otros autores han descrito con respecto a la efectividad de mepolizumab en este grupo etario. ${ }^{20,27}$

El perfil de seguridad observado en nuestros casos es consistente con lo que se ha descrito en la literatura, donde cefalea y nasofaringitis han sido los eventos reportados con más frecuencia, que afortunadamente sólo lo observamos en uno de los pacientes y fue relativamente fácil de tratar. ${ }^{28,29}$

Creemos que es importante incrementar nuestra experiencia con un mayor número de pacientes, más tiempo de seguimiento y también con otros anticuerpos monoclonales para fortalecer nuestro entendimiento de la caracterización de casos mexicanos con asma grave y su respuesta a estos nuevos tratamientos.

\section{CONCLUSIÓN}

Nuestra experiencia clínica inicial con mepolizumab en el tratamiento de dos adolescentes con asma grave eo- 
sinofílica refractaria no controlada ha sido favorable con reducciones importantes en las exacerbaciones de asma, mejoría del control del asma, disminución en la frecuencia de uso de medicamentos de rescate y mejoría en la función pulmonar de ambos. Esta experiencia inicial es un panorama que requiere ampliarse a más casos y por más tiempo para aportar evidencias de consistencia de resultados observados en otras poblaciones al contexto de pacientes mexicanos con asma grave.

\section{REFERENCIAS}

1. Global Strategy for Asthma Management and Prevention [Internet]. EUA: Global Initiative for Asthma; 2021. Available in: https:// ginasthma.org/gina-reports/

2. Menezes AM, Hallal P, Montes-de Oca M, Muino A, López-Varela, Talamo $C$ et al. Prevalence of asthma in Latin American middleaged and older adults and its overlap with diagnosis of COPD. Eur Respir J. 2011; 38 (Suppl 55): 4136.

3. Scichilone N, Barnes PJ, Battaglia S, Benfante A, Brown R, Canonica GW et al. The hidden burden of severe asthma: from patient perspective to new opportunities for clinicians. J Clin Med. 2020; 9 (8): 2397. Doi: 10.3390/jcm9082397.

4. Backman H, Jansson SA, Stridsman C, Eriksson B, Hedman L, Eklund BM et al. Severe asthma-a population study perspective. Clin Exp Allergy. 2019; 49: 819-828.

5. Guía Española para el manejo de Asma. GEMA 5.0.

6. McDonald VM, Kennington E, Hyland ME. Understanding the experience of people living with severe asthma. En: Chung KF, Israel E, Gibson PG, editores. Severe asthma. Reino Unido: European Respiratory Society; 2019.

7. Lane S, Molina J, Plusa T. An international observational prospective study to determine the cost of asthma exacerbations (COAX). Respir Med. 2006; 100 (3): 434-450. doi: 10.1016/j.rmed.2005.06.012.

8. Busse WW. Biological treatments for severe asthma: a major advance in asthma care. Allergol Int. 2019; 68 (2): 158-166. doi: 10.1016/j.alit.2019.01.004

9. Haldar P, Pavord ID, Shaw DE, Berry MA, Thomas M, Brightling CE et al. Cluster analysis and clinical asthma phenotypes. Am J Respir Crit Care Med. 2008; 178 (3): 218-224. doi: 10.1164/ rccm.200711-1754OC.

10. De Carvalho-Pinto RM, Cukier A, Angelini L, Antonangelo L, Maud $\mathrm{T}$, Rabe KF et al. Clinical characteristics and possible phenotypes of an adult severe asthma population. Respir Med. 2012; 106 (1): 4756. doi: 10.1016/j. rmed.2011.08.013.

11. Greenfeder S, Umland SP, Cuss FM, Chapman RW, Egan RW. Th2 cytokines and asthma. The role of interleukin-5 in allergic eosinophilic disease. Respir Res. 2001; 2 (2): 71-79. doi: 10.1186/rr41.

12. Holguin F, Cardet JC, Chung KF, Diver S, Ferreira DS, Fitzpatrick A et al. Management of severe asthma: a European Respiratory Society/American Thoracic Society guideline. Eur Respir J. 2020; 55 (1): 1900588. doi: 10.1183/13993003.00588-2019.

13. Partida-Gaytán A, Torre-Bouscoulet L, Macías MP, Raimondi A, Pizzichini E. Mepolizumab for the treatment of severe eosinophilic asthma. Revista Alergia México. 2020; 67: doi: doi.org/10.29262/ ram.v67i7.780.

14. Pavord ID, Korn S, Howarth P, Bleecker ER, Buhl R, Keene ON et al. Mepolizumab for severe eosinophilicasthma (DREAM): a multicentre, double-blind, placebo-controlled trial. Lancet. 2012; 380 (9842): 651-659. doi: 10.1016/S0140-6736(12)60988-X.

15. Ortega HG, Liu MC, Pavord ID, Brusselle GG, FitxGerald JM, Chetta A et al. Mepolizumab treatment in patients with severe eosinophilic asthma. N Engl J Med. 2014; 371 (13): 1198-1207. doi: 10.1056/ NEJMoa1403290.

16. Bel EH, Wenzel SE, Thompson PJ, Prazma CM, Keene ON, Yancey SW et al. Oral glucocorticoid-sparing effect of mepolizumab in eosinophilic asthma. N Engl J Med. 2014; 371 (13): 1189-1197. doi: 10.1056/NEJMoa1403291.

17. Chupp GL, Bradford ES, Albers FC, Bratton DJ, Wang-Jairaj J, Nelsen LM et al. Efcacy of mepolizumab add-on therapy on health-related quality of life and markers of asthma control in severe eosinophilic asthma (MUSCA): a randomised, double-blind, placebo-controlled, parallel-group, multicentre, phase $3 \mathrm{~b}$ trial. Lancet Respir Med. 2017; 5 (5): 390-400. doi: 10.1016/S22132600(17)30125-X.

18. Votto M, De Filippo M, Licari A, Marseglia A, De Amici M, Marseglia GL. Biological therapies in children and adolescents with severe uncontrolled asthma: a practical review. Biologics. 2021; 15: 133-142. doi: 10.2147/BTT.S252574.

19. Lin $\mathrm{CH}$, Cheng SL. A review of omalizumab for the management of severe asthma. Drug Des Devel Ther. 2016; 10: 2369-2378.

20. Yancey SW, Ortega HG, Keene ON, Bradford ES. Efficacy of addon mepolizumab in adolescents with severe eosinophilic asthma. Allergy Asthma Clin Immunol. 2019; 15: 53. doi: 10.1186/s13223019-0366-x.

21. Jocelyne Just $M D, P h D$, New perspectives of childhood asthma treatment with biologics,Pediatr Allergy Immunol. 2019; 30: 159171. doi: 10.1111/pai.13007.

22. Albers F C, Biologic treatment eligibility for real-world patients with severe asthma: the IDEAL study. Journal for Asthma. (Internet) 2018; 55 (2) 152-160. Available in: https://doi.org/10.1080/02770 903.2017.1322611.

23. Chung KF, Wenzel SE, Brozek JL, Bush A, Castro M, Sterk PJ, Adcock IM et al. International ERS/ATS guidelines on definition, evaluation and treatment of severe asthma. Eur Respir J. 2014; 43: 343-373.

24. Álvarez-Gutiérrez FJ, Blanco-Aparicio M, Plaza V, Cisneros C, García-Rivero JL, Padilla A et al. Documento de consenso de asma grave en adultos. Actualización 2020. Open Respir Arch. 2020; 2 (3): 158-174.

25. Chapman KR, Albers FC, Chipps B, Muñoz X, Devouassoux G, Bergna $M$ et al. The clinical benefit of mepolizumab replacing omalizumab in uncontrolled severe eosinophilic asthma. Allergy. 2019; 74 (9): 1716-1726. doi: 10.1111/all.13850.

26. Carpagnano GE, Pelaia C, D'Amato M, Crimi N, Scichilone N,Scioscia G, Resta O, Calabrese C, Pelaia G, Quarato CMI, Foschino Barbaro MP. Switching from omalizumab to mepolizumab: real-life experience from Southern Italy. Ther Adv Respir Dis. 2020; 14:1753466620929231. doi: 10.1177/17534666209231.

27. Hoshi M, Matsunaga M, Nogami K, Hamada K, Kobori T, Kainuma K, Nagao M, Fujisawa T. Three cases of severe adolescent asthma treated with mepolizumab: lung function trajectories. Asia Pac Allergy. 2020; 10 (2): e13. doi: 10.5415/apellergy.2020. 10.e13.

28. Lugogo N, Domingo C, Chanez P, Leigh R, Gilson MJ, Price RG et al. Long-term ef cacy and safety of mepolizumab in patients with severe eosinophilic asthma: a multi-center, open-label, phase IIlb study. Clin Ther. 2016; 38 (9): 2058-2070.e1. doi: 10.1016/j. clinthera.2016.07.010.

29. Khurana S, Brusselle GG, Bel EH, FitzGerald JM, Masoli M, Korn $S$ et al. Long-term safety and clinical bene $t$ of mepolizumab in patients with the most severe eosinophilic asthma: the COSMEX study. Clin Ther. 2019; 41 (10): 2041-2056. e5. doi: 10.1016/j. clinthera.2019.07.007

Conflicto de intereses: Armando Partida-Gaytán trabaja como gerente médico para GSK México. 